\title{
Potencialidades productivas del sector Agronegocios en la región Apurimac, Perú. Oportunidades para el Comercio Internacional
}

\author{
Martiniano Reyes-Olivo \\ mreyesoliv@gmail.com \\ Universidad Nacional Abierta-Venezuela \\ orcid.org/0000-0002-9337-046x
}

Rony Méndez Soto

ronymendezsoto@gmail.com

Universidad Nacional San Antonio Abad del Cusco

Orcid.org/0000-0003-1522 8756

\section{RESUMEN}

La región en estudio, ancestralmente, ha tenido como principal actividad económica la agricultura. Desde tiempos pre-hispánicos sus tierras fértiles han tenido vocación agrícola. En los últimos 20 años esta realidad cambió y se migró a actividades de minería de oro, plata y cobre principalmente. Si bien, los números macroeconómicos de PIB y Valores de exportación son importantes, esto no se ha traducido en un desarrollo endógeno local y produce poco valor agregado. De otra parte, se redujo paulatinamente el tamaño de la actividad agrícola de la región.

Este trabajo de investigación, tuvo como objetivo, conocer e identificar las potencialidades productivas del sector Agronegocios de la región Apurimac para valorar sus oportunidades de insertarse en la dinámica del comercio internacional apalancándose en sus ventajas comparativas de recursos naturales y su oferta agrícola andina. Se utilizó un enfoque cualitativo con una metodología de tipo documental a partir de fuentes de información primarias y secundarias, que permitieron recopilar y clasificar información con una temporalidad longitudinal entre los años 2017 y 2019 con un nivel de diseño no experimental.

Se encontraron fortalezas y oportunidades para afrontar un proceso de Agronegocios de exportación con bases sólidas, para lo cual se debe superar retos y desafíos tales como; la producción de auto consumo, la atomización de los productores, promover la asociatividad, además de fortalecer las cadenas de valor de Agroexportación que logren integrar la producción andina a los grandes centros de producción de la costa y norte peruano que permitan encarar con éxito un proceso de internacionalización.

Palabras Clave: Agroexportación, Agronegocios, Cadena de Valor, Carretera Interoceánica, Desarrollo Sustentable 


\title{
Productive potentialities of the Agribusiness sector in the Apurimac region, Peru: opportunities for international trade
}

\begin{abstract}
The region under study has, for centuries, had agriculture as its main economic activity. Since preHispanic times its fertile lands have had an agricultural vocation. In the last 20 years this reality changed and migrated to gold, silver and copper mining activities mainly. Although, the macroeconomic numbers of GDP and export values are important, this has not translated into local endogenous development and produces little added value. On the other hand, the size of the region's agricultural activity was gradually reduced.

The objective of this research was to understand and identify the productive potential of the Agribusiness sector in the Apurimac region, in order to assess its opportunities for insertion into the dynamics of international trade, leveraging its comparative advantages in natural resources and its Andean agricultural supply. A qualitative approach was used with a document-type methodology based on primary and secondary information sources, which allowed the collection and classification of information with a longitudinal temporality between 2017 and 2019 with a nonexperimental design level.
\end{abstract}

Strengths and opportunities were found to face a process of Agribusiness export with solid bases, for which it is necessary to overcome challenges such as; the production of self-consumption, the atomization of the producers, to promote the associativity, besides strengthening the chains of value of Agro export that manage to integrate the Andean production to the big centers of production of the coast and Peruvian north that allow to face successfully a process of internationalization

Key Words: Agribusiness, Agroexport, Interoceanic Road, Value Chains, Sustainable Development,

Artículo recibido: 03 nov. 2020 Aceptado para publicación: 07 dic. 2020 Correspondencia mreyesoliv@ gmail.com Conflictos de Interés: Ninguna que declarar 


\section{INTRODUCCIÓN}

Actualmente uno de los rubros más demandados a nivel global, es el de los alimentos, bien sea, en forma de alimentos procesados (industriales) o los productos de origen agrícola. En tal sentido, el crecimiento de la población mundial, ha traído consigo nuevos desafíos y oportunidades en el contexto del comercio internacional. Los primeros nos plantean interrogantes como la siguiente: ¿Cómo alimentar a una población mundial que crece aritméticamente mientras que la producción de alimentos crece geométricamente? Según estimaciones de la Organización de las Naciones Unidas para la Alimentación y la Agricultura, FAO (2009) se calcula que en el año 2050 el planeta contará con 9.500 millones de habitantes, 35\% más de la población actual, las cuales demandarán un $70 \%$ más de alimentos. (Larrea, et. al, 2018)

En cuanto al paradigma de las oportunidades, existen ventajas comparativas para la región Latinoamericana, la cual posee un tercio de los recursos de agua dulce del planeta, más que cualquier otra región en desarrollo, si se miden sobre una base per cápita. También cuenta con cerca del 28\% de la tierra del mundo -que ha sido identificada con potencial mediano a alto- para la expansión sostenible de áreas cultivables. En su conjunto, ya es la región que registra la mayor exportación neta de alimentos del mundo, y hasta el momento solo ha alcanzado una pequeña parte de su potencial para ampliar la producción agrícola dirigida al consumo regional y la exportación mundial. (BID 2014)

En lo que al Perú concierne y su posición dentro de este escenario, vemos que ocupa el primer lugar entre los países con mayor crecimiento agroexportador de la región Latinoamericana con tasas anuales promedio entre el 15\% y 20\%. Vale decir que el sector Agronegocios se ha constituido, en las dos últimas décadas, en la segunda actividad económica con mayor rentabilidad después de la minería, multiplicándose casi diez veces sus ganancias a nivel de exportaciones de productos no tradicionales por un valor de $\$ 13.791$ millones que representan un $8 \%$ del PIB nacional. El crecimiento de este sector viene dado por tres factores fundamentales: a) La apertura de la economía peruana a partir de la década de los años 90'con promoción y apoyo del Estado para el sector agrícola b) La suscripción del Perú a Tratados de Libre Comercio (TLC), de Acuerdos Regionales y Bilaterales de Comercio Internacional c) Flexibilización de las condiciones laborales y fiscales para el sector agroexportador.

Según el último Censo Nacional Agropecuario (INEI-CENAGRO 2012) señala que el 60\% de la Agroexportación se focalizan en la región de la costa, mientras que el mayor número de unidades 
agropecuarias - cuantificadas en un 64\%- se encuentran en la sierra. Esta concentración se debe principalmente a las facilidades que ofrecen los proyectos de irrigación y la infraestructura vial disponible. (Cheng y Pintado, 2015).

Al referirnos a la región de Apurímac, debemos saber que su nombre proviene de un vocablo quechua que etimológicamente significa: $\mathrm{Apu}=\mathrm{Dios}$ y Rímac= Hablador, es decir, "Dios que Habla”. Esta hermosa región, forma parte, a su vez, de la denominada región macro sur del Perú, la cual está integrada -además de Apurímac- por seis departamentos más, a saber: Arequipa, Cusco, Madre Dios, Moquegua, Puno y Tacna, para un total de siete departamentos. La región macro sur, posee grandes potencialidades en lo que tiene que ver, con su inventario de recursos naturales; la biodiversidad, sus tierras fértiles y el agua abundante. La región macro sur goza de una excelente ubicación geográfica, que le aproxima a grandes mercados extranjeros representados por Bolivia, Brasil y Chile, a los cuales se puede accesar por vía terrestre a través de la carretera Interoceánica. Las poblaciones foráneas más importantes -por su tamaño y capacidad comercial- que colindan con la referida región son; los estados de Acre, Rondonia y Mato Grosso (Centro occidente brasileño) y Arica-Tarapacá (Norte de Chile). Para efectos de este trabajo investigativo, nos centraremos en los mencionados estados del Brasil. Todo lo anterior representa un mundo de oportunidades para el impulso del comercio internacional de la región macro sur en su conjunto y en especial para Apurimac.

La región en estudio, históricamente ha tenido como principal actividad económica la agricultura, es así, como desde tiempos pre-hispánicos sus tierras fértiles han tenido marcada vocación agrícola, teniendo como protagonista a la importante civilización de los legendarios Chancas quienes posteriormente sucumbieron ante la dominación del Imperio Inca, bajo el reinado de Pachacutec. Con la llegada de los españoles, se introdujo la actividad pecuaria, trayendo nuevas especies animales como el ganado bovino, equino, ovino y porcino que se adaptaron adecuadamente al medioambiente y se sumaron a las especies de los camélidos las cuales eran originarias de la zona andina del Perú, constituyéndose en las actividades económicas fundamentales durante largos siglos en la región. (INEI, 2000)

Hacia finales de la década de los noventa del siglo pasado, comenzó la explotación minera en el sur andino peruano, principalmente en los departamentos de Arequipa y Cusco. Más tarde, con el inicio del nuevo siglo, comenzaría la extracción y comercialización internacional -a gran escala- de la minería en la región de Apurimac. Esta actividad ha venido desplazando a nivel de importancia 
económica, a la ancestral agricultura de productos andinos así como también la producción pecuaria. En la actualidad, según cifras de (INEI, 2020) la dinámica productiva de la minería aporta el $61 \%$ del PIB de la región, por su parte la producción agropecuaria suma un 6\% al PIB.

Estas dos realidades se contrastan claramente. Mientras la actividad agrícola y pecuaria, es de tipo extensivo, lo que permite generar más empleo productivo, tanto directo como indirecto, favorece la asociatividad y fortalece las economías locales, creando riqueza y recursos que impactan en la redistribución del ingreso regional, por su parte, la actividad minera es típicamente una actividad intensiva, que requiere menor mano de obra - por su alto grado de tecnificación- en comparación con la agricultura. Además, su orientación es de exportación hacia mercados extranjeros, dejando muy poco valor agregado en la región.

El objetivo de esta investigación se focaliza en conocer e identificar las potencialidades productivas en el sector Agronegocios de la región Apurimac con una perspectiva exploratoria para evaluar oportunidades de inserción de la economía regional en la dinámica del comercio internacional apalancándose en sus ventajas comparativas de recursos naturales y su oferta agrícola andina, dentro de un proceso con una visión más inclusiva y con un mayor impacto socio-económico en la población Apurimeña.

\section{MATERIAL Y MÉTODOS}

La presente investigación se llevó a cabo bajo un enfoque cualitativo con una metodología de revisión de la literatura tipo narrativa a partir de fuentes de información primaria y secundaria, obtenidas a través de bases de datos como Scielo, Google Académico y Latíndex, así como repositorios universitarios, bibliotecas digitales institucionales y revistas especializadas on line que permitieron recopilar y clasificar información con una temporalidad longitudinal entre los años 2017 y 2019 con un nivel de diseño no experimental que tuvo por objetivo primordial conocer e identificar las potencialidades en el sector Agronegocios para la exportación de productos no tradicionales de la región Apurimac como una oportunidad de inserción en la dinámica del comercio internacional. Asimismo, se determinaron las características geográficas y climáticas de la región, los productos agrícolas nativos y su potencialidad comercial, así como también se identificaron las fortalezas y debilidades del sector Agronegocios regional para luego observar las tendencias y oportunidades de los mercados foráneos, específicamente los estados centro occidentales del vecino Brasil; acre, Rondonia y Mato Grosso, los cuales colindan con la región macro sur peruana de la cual el departamento de Apurimac forma parte. Finalmente, se plantearon estrategias para direccionar los 
objetivos de comercialización internacional en el negocio agroalimentario. En cuanto a la información estadística que respalda este trabajo, algunas de ellas se obtuvieron partiendo de diferentes bases de datos del Ministerio de Comercio Exterior y Turismo- Promperu (MINCETUR), Ministerio del Trabajo y Promoción del Empleo (MTPE), Instituto Nacional de Estadística (INEI), Instituto Brasileño de Geografía e Informática (IBGE), la Organización de las Naciones Unidad para la agricultura y la alimentación (FAO). Y otras fueron, procesadas a través del paquete Office 2016: procesador de palabras Word y hojas de cálculo Excel, bajo programa Windows 10

\section{RESULTADOS}

\section{Superficie y División Territorial}

El departamento de Apurímac fue fundado el 28 de abril de 1873. Se ubica en la parte sur oriental de la República del Perú. Su extensión territorial es de 20,895.8 Km2, que representa el $1.63 \%$ del territorio nacional. Sus límites son: por el Norte con los Departamentos de Ayacucho y Cusco, por el Este con el Departamento del Cusco, por el Sur con los Departamentos de Arequipa y Ayacucho, por el Oeste con el Departamento de Ayacucho. En cuanto a su división política tiene un total de 7 provincias y 80 distritos.

\section{Aspectos Geográficos y climáticos de la Región}

Su territorio es accidentado, valles profundos y agrestes cumbres, alternan con altas mesetas o punas y picos nevados. El clima es variado de acuerdo a los pisos de altitud. Cálido y húmedo en el fondo de los cañones profundos del Apurimac, Pampas y Pachachaca templado y seco en las altitudes medias. Frío y con acentuada sequedad atmosférica en la alta montaña y muy frío en las cumbres nevadas. La variación de la temperatura es muy significativa y aumenta con la altitud. Las precipitaciones son abundantes de diciembre a abril y el periodo seco, con lluvias escasas de mayo a noviembre. En la época de lluvias, los huaycos, inundaciones y derrumbes son fenómenos de gran frecuencia y afectan constantemente las vías de comunicación. (PERX- Apurimac, 2006)

\section{Recursos Naturales. Suelos y Agua}

El departamento cuenta con una superficie de tierras de 2’089,579 hectáreas (Ha), que representan el $1.62 \%$ del total nacional (Perú 128,521,560 Ha.). El mayor porcentaje de superficie corresponde a tierras de protección, seguidas de tierras de uso agrícola, dónde los pastos representan el 62.1\%, le sigue las tierras protegidas con $20.1 \%$, los montes y bosques representan el $9.1 \%$ y, las tierras en riego y secano solo el $8.7 \%$. 
La Región cuenta con un extraordinario potencial hídrico, el caudal de agua que circula por el sistema proviene principalmente de aguas superficiales, de las precipitaciones pluviales, deshielos de glaciares y nevados, que forman y dan origen a ríos con caudales permanentes. El drenaje hidrográfico en el territorio de este departamento tiene una orientación general de Sur a Norte y todos sus ríos pertenecen a la cuenca del río Apurímac, al que dan sus aguas por la margen izquierda. Indicadores sociales y económicos de la región Apurímac

A continuación, se muestran los indicadores socio-económicos básicos de la región Apurimac.

\section{Tabla $\mathbf{N}^{\circ}$ 1:}

\section{Tabla $\mathrm{N}^{\circ} \mathbf{1}$}

Indicadores socio-económicos de la región Apurímac

Población

PEA*

Empleo

Pobreza

PBI Minería

PBI

agropecuario

Analfabetismo

$\mathrm{IDH}^{*}$
405759 Habitantes

267881 personas

Tasas: Empleo 35\%, Desempleo 3.8\%, Sub-empleo 62\%

Pobreza: 35\%, Pobreza extrema: 5.6\%

$61.70 \%$

$6.50 \%$

Mujeres: 22.6\%, Hombres: 5.6\%

Lugar $\mathrm{N}^{\circ} 22^{\circ}$ de 24 regiones evaluadas

Tabla $N^{\circ}$ 1. Fuente: Elaboración propia sobre la base de Mincetur 2019 / INEI (2019)

Nota: * Población Económicamente Activa / * Índice de Desarrollo Humano

Llama la atención la cifra de sub-empleo de la Población Económicamente Activa (PEA), tomando en cuenta el tamaño de la población, el cual es relativamente pequeña y la alta tasa de analfabetismo de las mujeres en la región.

\section{Perfil de los principales agros productos de la región de Apurimac. Oferta exportable.}

La cultura ancestral de los habitantes de los andes peruanos le permitió desarrollar técnicas como los cultivos en andenes y riego por goteo. A lo largo de los siglos y dada las condiciones climáticas especiales y la fertilidad de la tierra, en el Perú se han logrado producir agro productos únicos en el planeta por su alto valor nutritivo y medicinal con características inigualables, los cuales han sido denominados los "Superalimentos" y están llamados a ser una oportunidad apreciable para dar a conocer al mundo y contribuir a la seguridad alimentaria de la humanidad. (Promperu, 2019) A 
continuación, una relación de los principales de ellos, ver Tabla $\mathrm{N}^{\circ} 2$

Tabla $\mathbf{N}^{\circ} \mathbf{2}$

Oferta exportable de agro productos andinos

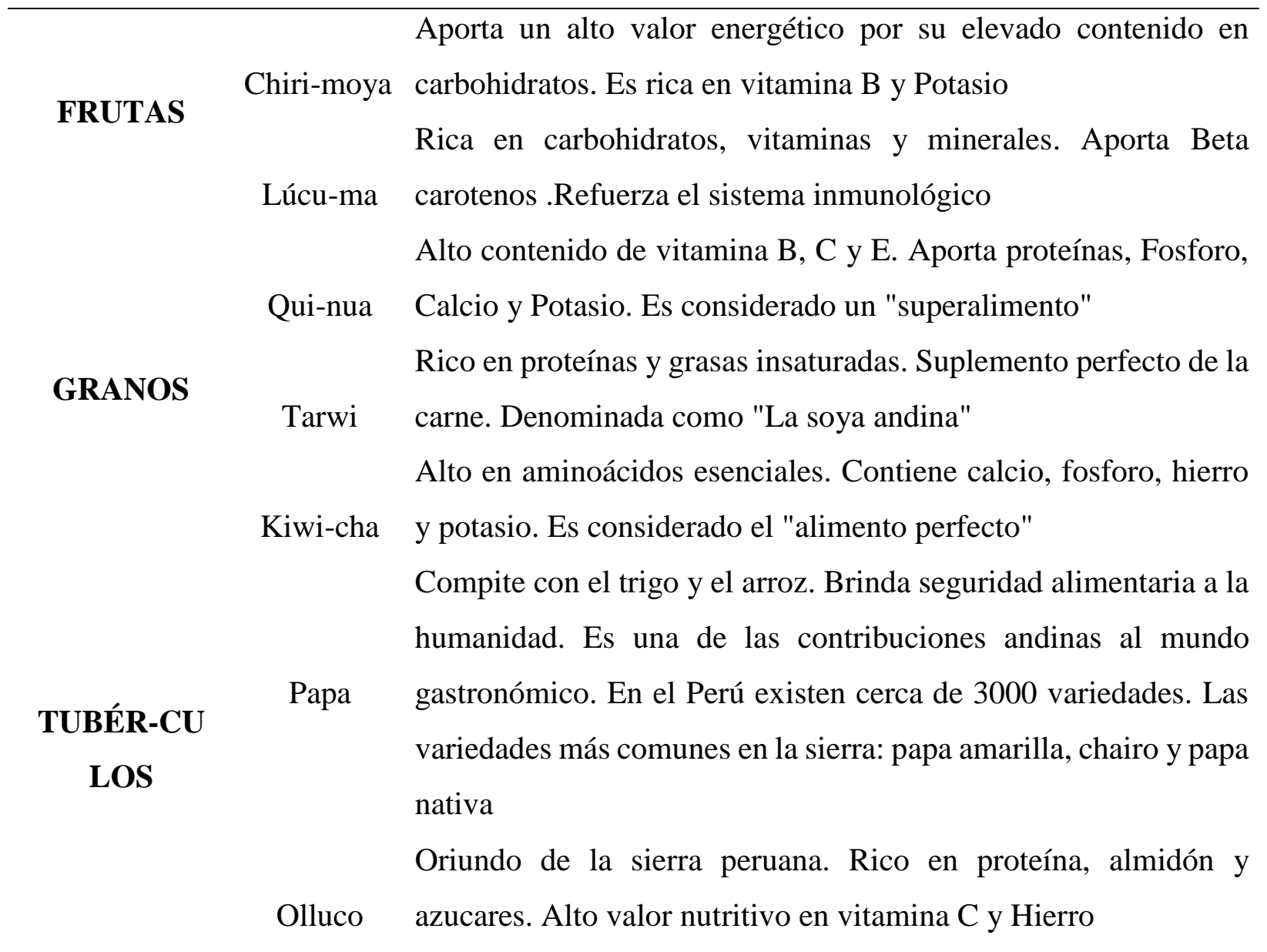

Tabla $N^{\circ}$ 2. Fuente: Ministerio de Agricultura y Riego (MINAGRI, 2019)

\section{Análisis FODA de la competitividad exportadora de la región Apurímac}

Cuando se analizan las oportunidades de negocios internacionales para la exportación, es básico conocer las posibilidades reales de alcanzar dicha meta, sobre la base de las fortalezas y debilidades con que se cuenta, así como también, de las oportunidades y amenazas que existen en el entorno, todo lo cual es determinante para visualizar el éxito de las actividades. Al estudiar el perfil del sector agroexportador de la región, se encontraron, a nivel de competitividad, las características que se muestran en la Tabla $\mathrm{N}^{\circ} 3$ : 
Tabla $\mathbf{N}^{\circ} \mathbf{3}$

Análisis FODA sector Agronegocios región Apurímac

\section{Fortalezas}

Biodiversidad

Estacionalidad

Diversidad climática

Recursos Hídricos abundantes

Grandes extensiones cultivables

Conocimientos ancestrales

Mano de Obra

Debilidades

Fragmentación de tierras

Infraestructura deficiente

Bajo nivel tecnológico

producción para autoconsumo

Necesidad de Capacitación y certificación

Débil Logística internacional

\section{Oportunidades}

Tratados Comerciales

Carretera Interoceánica

Productos de fama mundial

Cercanía de mercados destinos

Productos Orgánicos y no tradicionales

Alta demanda

Transporte Internacional

Amenazas

Cambios climáticos

Competencia internacional

Barreras para-arancelarias

Monopolios y tractores comerciales

Conflictos sociales y políticos

Terrorismo y narcotráfico

\section{Tabla $N^{\circ} 3$. Fuente: Elaboración propia}

Es claro que la región posee grandes fortalezas que generan mayores oportunidades, pero ello va a depender de cómo se vayan atenuando las debilidades para enfrentar de mejor manera las amenazas del entorno. Esta oferta exportable, deberá ser validada con instrumentos mercadotécnicos en posteriores estudios para determinar su factibilidad.

\section{Indicadores macroeconómicos de los mercados destinos brasileros.}

Conocer los mercados destinos, es tarea fundamental para preparar las estrategias y diseñar los objetivos; comerciales, de mercadotecnia y logísticos. Para ello, una herramienta fundamental son los indicadores macroeconómicos que sirven para identificar el perfil socio-económico y comercial de los mismos.

Estos son los datos que se encontraron de los mercados brasileros vecinos, los cuales serían destinos comerciales en una primera etapa. Ver Tabla $\mathrm{N}^{\circ}$ 4. Vale destacar que estos estados del norte de Brasil están más cerca de la macro región que de la propia capital de Brasil, Brasilia: 


\section{Tabla $\mathbf{N}^{\circ} 4$}

\section{Indicadores macroeconómicos del centro occidente brasileño}

\begin{tabular}{|c|c|c|c|c|}
\hline & & PIB per & & Extensión \\
\hline Estado & Población & cápita & IDH & (KM2) \\
\hline Acre & 894740 & $\$ 2839$ & 0,716 (alto) & 164122 \\
\hline Rondonia & 1796640 & $\$ 3391$ & 0,725 (alto) & 238513 \\
\hline Mato Grosso & 3526220 & $\$ 3393$ & 0,725 (alto) & 903207 \\
\hline
\end{tabular}

Como se puede observar, solo tres estados brasileños, representan un tamaño de mercado de más de 6 millones de habitantes- mayor que la población de la macro región sur en su totalidad- los cuales son potenciales consumidores de los agro productos de la región, con un promedio de ingresos per cápita de $\$ 3393$.

\section{Carretera Interoceánica. Una vía hacia el progreso de la Macro Región Sur Peruana}

La carretera Interoceánica, constituye una gran ventana comercial para apuntalar el desarrollo de la región macro sur como un todo y en especial para la región Apurímac. La Interoceánica no sólo genera nuevos mercados para la oferta agroexportadora actual de Apurimac, sino también la demanda de productos que aún no oferta la región, pero que tiene potencial para desarrollarlos, tales como; servicios de mantenimiento mecánico de unidades de transporte internacional de carga, suministro de combustible y lubricantes, servicios aduaneros y logísticos, almacenaje, seguros, etc. En su conjunto estas variables, significan la creación de empleo productivo, directo e indirecto, generación de valor agregado local, desarrollo del campo y su productividad, incremento de la asociatividad productiva y comercial, en suma, mejoras en el nivel de ingresos de la región y, por ende, de su calidad de vida.

Es de destacar que, la distancia que existe entre Andahuaylas y la región fronteriza de Iñapari (Brasil) es de 1.179,95 km apenas, lo que representa un viaje terrestre relativamente cercano de 24 horas de tránsito, aproximadamente. Es decir, los productos de la región están a un día de viaje hasta la entrada comercial de Brasil- para ser colocados en ese gran mercado vecino. Igualmente, esta vía ofrece la posibilidad de conectar a la región macro sur del Perú con el océano Atlántico a través del principal puerto de Brasil -puerto de Santos- de cara hacia los mercados de África, y Asia 
con consumidores potenciales que representan el 70\% de la población mundial. (MINCETUR, 2017)

\section{DISCUSION}

Los resultados son bastante claros para afirmar que existen grandes oportunidades para el desarrollo de los Agronegocios de exportación en la región. Por supuesto que la tarea no es nada fácil dadas las condiciones actuales, donde se privilegia la actividad económica extractiva de materias primas dirigidas a la exportación, como lo el oro, plata y cobre, lo cual ha venido a trastocar toda una forma de vida milenaria representada por las actividades agrícolas andinas. Así vemos como la industria extractiva de minerales, arroja cifras macroeconómicas como estas; desde el 2015 al 2019 el PIB de la región se ha triplicado al pasar de $\$ 1100$ millones a $\$ 3000$ millones con un aporte del $61 \%$ mientras que el PIB que aporta actividad agrícola y pecuaria, en los mismos años, decayó de 14.9\% a un 6,5\%, es decir, redujo su tamaño en más de un 50\%. Ver tabla $\mathrm{N}^{\circ}$ 5. Paradójicamente, la tasa de desempleo de la región presenta un incremento de $3.1 \%$ a 3.8\% en los años referidos, mientras que la tasa de informalidad y sub-empleo alcanzaron para el año 2018, $88 \%$ y 62\%, respectivamente. (MTPE, 2019).

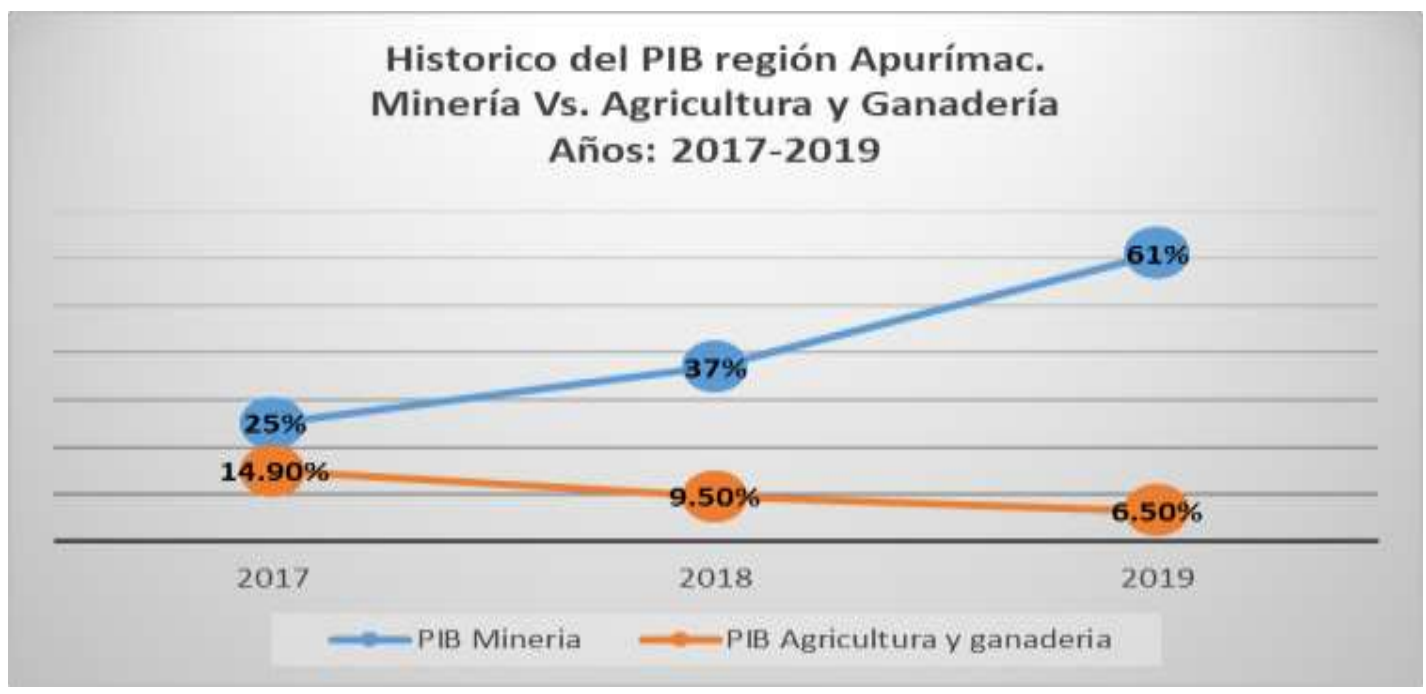

Tabla N5. Fuente: Elaboración propia sobre la base de datos de INEI. 2019

Este panorama, sugiere una profunda contradicción del modelo económico que impera en la región y que deja al desnudo una cruda realidad: la actividad extractiva de materias primas arroja grandes números macroeconómicos que no impactan directamente en la calidad de vida del Apurimeño porque aporta muy poco valor agregado local. Los beneficios llegan de manera indirecta -vía Canon minero- hacia el gobierno central que luego son redistribuidos -aguas abajo- a los otros niveles de gobierno. De esta forma, por un lado, se profundiza la explotación minera, con concesiones a 
empresas trasnacionales a 30 y 40 años de vigencia, mientras las actividades productivas autóctonas de la región se vienen gradualmente a menos.

La otra cara de la moneda -de manera contrastante- nos muestra que el Perú en la actualidad, se ha convertido en uno de los grandes productores y exportadores de agro alimentos del mundo, ocupando el primer lugar entre los productores de espárragos, arándanos y quinua, los segundos en mandarinas, terceros en palta y aceitunas, cuartos en palmito y sus derivados y quintos en producción de uvas frescas, lo que demuestra que hay dos realidades económicas distintas dentro del mismo país y la pregunta que cabe sería; ¿Porque no integrar y consolidar a todas las regiones productivas del país en una misma sinergia y dinámica de comercio internacional? De tal manera que, la carretera interoceánica significa una gran oportunidad para impulsar inversiones para el desarrollo de la región sur del Perú que permitan apalancar la integración económica nacional e internacional. Siendo las zonas fronterizas, las del centro occidente del Brasil (Estados de Acre, Rondonia, Mato Grosso) y del norte de Chile (Arica-Tarapacá) los mercados más importantes, por su tamaño y capacidad comercial.

Si a esto le agregamos que las mercancías provenientes de la región macro sur peruana pueden utilizar operativamente el principal puerto de Brasil -puerto de Santos- ubicado en el lado Atlánticocomo trampolín para alcanzar a los mercados de África y Asia, en términos económicos, esto puede convertirse en una puerta de entrada para el desarrollo sustentable de Apurimac o simplemente ser una oportunidad perdida en el tiempo, que nos dejará rezagados en el camino del comercio internacional y nos alejará del tan anhelado crecimiento económico. En ese sentido, la carretera Interoceánica también ofrece la oportunidad de recuperar la integración del sur del Perú con el mundo como lo hicieran los incas y posteriormente lo acentuaran los españoles.

\section{CONCLUSIONES}

Finalmente, podemos concluir que el principal reto que tiene la región es el de retomar la senda de la producción agrícola -como actividad económica alternativa- basada en una visión renovada de Agronegocios de exportación, preferiblemente por vía directa, como una opción valedera que permita superar la estructura económica semi-rural, la generación de empleo, la producción de auto consumo y la atomización de los productores favoreciendo la asociatividad, además de fortalecer las cadenas de valor de Agroexportación que logren integrar la producción andina a los grandes centros de producción que se dan en la costa y en el norte del país. Las estrategias deben centrarse en enfoques técnicos-productivos a mediano y largo plazo más que una visión política cortoplacista. 
Para ello, como se propone en el trabajo del propio (PromPerú, 2015) existe una necesidad perentoria de conjugar a las fuerzas vivas del país y de la región alrededor de metas comunes que permitan amalgamar al Estado como ente regulador y promotor, al empresariado privado representado en las organizaciones de comercio exterior como ADEX, COMEX, Cámaras de Industria, Comercio y Turismo que hagan presencia activa en la macro región sur y por supuesto, a la Academia, representadas por las universidades nacionales y privadas, en su papel de capacitador-formador-investigador y asesor. El gran desafío del sur peruano radica en vencer su actual fragmentación espacial, social y económica. De otro lado, la región tiene enormes posibilidades de convertirse en la gran bisagra económica entre el gigantesco mundo asiático en expansión y este eje sur peruano, boliviano y brasileño. (PUCP, 2019)

\section{REFERENCIAS BIBLIOGRÁFICAS}

Banco Interamericano de Desarrollo (BID) 2014. La Próxima Despensa Global. Pág. N ${ }^{\circ}$. Recuperado de: https://publications.iadb.org/publications/spanish/document/Lapr\%C3\%B3xima-despensa-global

Cheng, G. y M. Pintado. 2015. El boom agroexportador, pero ¿de qué productores? La Revista Agraria, 171:13-15.

Instituto Nacionales de Estadística e Informática (INEI) 2012. IV Censo Nacional Agropecuario (CENAGRO)

Instituto Nacional de Estadística e Informática (INEI) (2000). Conociendo a Apurímac. Pag. 8. Recuperado de: https://www.inei.gob.pe/media/MenuRecursivo/publicaciones_digitales/ Est/Lib0441/Libro.pdf

Instituto Nacional de Estadística e Informática (INEI) 2020. Valor Agregado Bruto por actividades Económicas región Apurimac. 2007-2019. Recuperado de: https://www.inei.gob.pe/estadisticas/indice-tematico/producto-bruto-interno-pordepartamentos-9089/

Instituto Nacional de Estadística e Informática (INEI) Censo Nacional, XII de Población, VII de Vivienda y III de Comunidades Indígenas 2017. Recuperado de: http://censo2017.inei.gob.pe/poblacion-del-departamento-de-apurimac-totalizo-405-mil759-personas-al-2017/ 
Larrea, H., Ugaz, C. y Flórez, M. (2018) El Sistema de Agronegocios del Perú: De la agricultura Familiar al negocio agroalimentario. Revista Mexicana de Agronegocios. Vol. 43. Pag. 3. ISSN: 1405-9282. Recuperado de: http://www.redalyc.org/articulo.oa?id=14158242001

Ministerio de Comercio Exterior y Turismo (MINCETUR) 2006. Plan Estratégico Regional de Exportación Región Apurímac (PERX)-Apurímac. Recuperado de: https://cdn.www.gob.pe/uploads/document/file/354668/PERX_APURIMAC-2.pdf

Ministerio de Comercio Exterior y Turismo (MINCETUR) 2017. Guía de Rutas Terrestres a

Brasil. Programa Consolida Brasil. Recuperado de:

http://www.siicex.gob.pe/siicex/portal5ES.asp?_page_=855.82900

Ministerio de Comercio Exterior y Turismo. Promperu 2019. Exportaciones del Perú. 2019.

Recuperado de: http://www.siicex.gob.pe/siicex/portal5ES.asp?_page_=975.42200

Ministerio del Trabajo y Promoción del Empleo (MTPE) (2019). Región Apurímac: Panorama

Laboral. Lima. Recuperado de: https://cdn.www.gob.pe/ uploads/document/file/362 518/Panorama_Laboral_-_2018.pdf

Organización de las Naciones Unidas para la Alimentación y la Agricultura (FAO) (2009). La agricultura mundial en la perspectiva del año 2050. FAO: Roma. Recuperado de http://www.fao.org/fileadmin/templates/wsfs/docs/Issues_papers/Issues_papers_SP/

La_agricultura_mundial.pdf

Organización de las Naciones Unidas para la Alimentación y la Agricultura (FAO) (2014) La Próxima Despensa Global. Como América Latina Puede Alimentar al Mundo. Recuperado de: $\quad$ https://publications.iadb.org/publications/spanish/document/La-pr\%C3\%B3ximadespensa-global-C\%C3\%B3mo-Am\%C3\%A9rica-Latina-puede-alimentar-al-mundo-

Pontificia Universidad Católica del Perú. (PUCP) Instituto de Estudios Internacionales. Serie Política Exterior y Relaciones Internacionales. Intereses del Perú en la Región Sur. Recuperado de: $\quad$ http://repositorio.pucp.edu.pe/index/bitstream/handle/123456789 1134496/2018\%20Intereses\%20del\%20Per\%C3\%BA\%20en\%20la\%20Regi\%C3\%B3n\%2 OSur.pdf? sequence $=1$ \&isAllowed $=y$

Promperu. 2019 Catálogo de Super food peruanos. Recuperado de: https://issuu.com/promperu/docs/catalogo_superfoods_espa_ol_1_

PromPerú. (2015) Análisis de Alternativas para la utilización de la Carretera Interoceánica Sur.

Recuperado de: http://repositorio.promperu.gob.pe/handle/123456789/1788 